\title{
New Avenues for Old Travellers: Phenotypic Evolutionary Trends Meet Morphodynamics, and Both Enter the Global Change Biology Era
}

\author{
Davide Tamagnini $^{1,2} \mathbb{D} \cdot$ Daniele Canestrelli $^{3} \mathbb{D} \cdot$ Carlo Meloro $^{4} \mathbb{D} \cdot$ Pasquale Raia $^{5} \mathbb{D} \cdot$ Luigi Maiorano $^{1,2} \mathbb{D}$
}

Received: 25 January 2021 / Accepted: 22 July 2021 / Published online: 16 August 2021

(c) The Author(s) 2021

\begin{abstract}
Evolutionary trends (ETs) are traditionally defined as substantial changes in the state of traits through time produced by a persistent condition of directional evolution. ETs might also include directional responses to ecological, climatic or biological gradients and represent the primary evolutionary pattern at high taxonomic levels and over long-time scales. The absence of a well-supported operative definition of ETs blurred the definition of conceptual differences between ETs and other key concepts in evolution such as convergence, parallel evolution, and divergence. Also, it prevented the formulation of modern guidelines for studying ETs and evolutionary dynamics related to them. In phenotypic evolution, the theory of morphodynamics states that the interplay between evolutionary factors such as phylogeny, evo-devo constraints, environment, and biological function determines morphological evolution. After introducing a new operative definition, here we provide a morphodynamics-based framework for studying phenotypic ETs, discussing how understanding the impact of these factors on ETs improves the explanation of links between biological patterns and processes underpinning directional evolution. We envisage that adopting a quantitative, pattern-based, and multifactorial approach will pave the way to new potential applications for this field of evolutionary biology. In this framework, by exploiting the catalysing effect of climate change on evolution, research on ETs induced by global change might represent an ideal arena for validating hypotheses about the predictability of evolution.
\end{abstract}

Keyword Biological rule $\cdot$ Non-Brownian evolution $\cdot$ Phenotype $\cdot$ Climate change biology $\cdot$ Ecomorphology

\section{History and Future of Evolutionary Trends}

Evolutionary trends (ETs) are traditionally defined as persistent and directional changes in the state of one or more quantitative traits, resulting in substantial changes through

Davide Tamagnini

davide.tamagnini@uniroma1.it

1 Department of Biology and Biotechnologies "Charles Darwin", University of Rome "La Sapienza", Rome, Italy

2 Museum of Zoology, Sapienza Museum Centre, University of Rome "La Sapienza", Building, Viale dell'Università 32, 00185 Rome, Italy

3 Department of Ecological and Biological Science, University of Tuscia, Viterbo, Italy

4 Research Centre in Evolutionary Anthropology and Palaeoecology, School of Biological and Environmental Sciences, Liverpool John Moores University, Liverpool, UK

5 Department of Earth, Environmental and Resources Sciences, University of Naples "Federico II", Napoli, Italy time and representing the primary phenomenon characterising evolution at high taxonomic levels and over long-time scales (Gould, 2002; McNamara, 2006; McShea, 2005). Alroy (2000) described the study of ETs as "one of the oldest and more intriguing topics in evolutionary biology", agreeing with McKinney (1990), who indicated the concept of ET as "arguably the single most important in the study of evolution".

The quest for ETs started early in macroevolution and quickly assumed crucial importance in phenotypic evolution and many other fields of evolutionary biology. The first groundbreaking investigations concerning phenotypic directional evolution led to the description of iconic and pervasive trends throughout the history of a single clade, frequently relying on the evidence available from fossil record (e.g., increasing hypsodonty and gradual acquisition of the monodactyl posture in equids caused by the spread of grasslands - Kowalewsky, 1874). Then, the formulation of the so-called 'biological rules' extended the definition of ETs to include directional responses to ecological, climatic 
or biological gradients, with several clades being simultaneously analysed obtaining the same evolutionary pattern (e.g., latitudinal or elevation gradients, like in Allen's or Bergmann's rules - Allen, 1877; Bergmann, 1847).

During the second half of the twentieth century, an animated debate rose regarding how to identify an ET. In particular, a key point in this debate was whether to find out a directional pattern in evolution suffices to establish the presence of an ET, or if an explanation about the underpinning biological mechanisms is needed (Dayan \& Simberloff, 1998; Mayr, 1956; Meiri, 2011). Further theoretical battlegrounds concerned erroneous interpretations of processes involved in the occurrence of directional evolution. In the past, these misinterpretations led to gross oversimplifications of the complexity of evolutionary dynamics and produced "common portrayals of evolution in non-academic settings including outright notions of 'advancement', that held sway within evolutionary biology as well" (Gregory, 2008 , p. 259). For example, Edward D. Cope based his concept of kinetogenesis (i.e., theory stating that movements of animals aided in the alteration and development of moving parts of the body) on the idea that key evolutionary novelties originate from generalized phenotypes, in keeping with Lamarck's view of evolution as possessing intrinsic directionality (Bowler, 1977). Whereas kinetogenesis (and its allied concept of orthogenesis) became extinct, the idea that evolution may possess a weak form of directionality (i.e., in contrast to old views of evolution as a movement towards 'perfection of life') in specific circumstances recently found support (Raia \& Fortelius, 2013; Raia et al., 2016). This vision relies on the concept that specialisation and adaptation towards optimal phenotypes can only occur after the birth of clades, for example, originating directional trends through time (Ayala, 1988; Gould, 1988a; Raia \& Fortelius, 2013; Raia et al., 2016; Rosenzweig \& McCord, 1991). Similar weak forms of directionality were suggested to occur in specific fields of evolutionary biology, such as the evolution of organismal diversity and complexity. For example, the zero-force evolutionary law (McShea \& Brandon, 2010; McShea et al., 2019) stated that "in any evolutionary system in which there is variation and heredity, there is a tendency for diversity and complexity to increase, one that is always present but may be opposed or augmented by natural selection, other forces or constraints acting on diversity or complexity" (McShea et al., 2019, p. 1103).

Although the search for new ETs and the validation of the existing ones remain central topics in evolutionary biology (Cardini, 2019; Cardini \& Polly, 2013), the formulation of a well-supported and operative definition of ET remains an unsolved issue. The lack of a broadly accepted operative definition prevented evolutionary biologists from unanimously pinpointing the requirements needed for detecting and validating ETs and blurred the definition of conceptual differences between ETs and other independent/ nested key concepts in evolution, whose operative definition had already been formulated (e.g., convergence - Stayton, 2015a, 2015b).

The pivotal importance of ETs in evolutionary biology can be understood by considering many possible contexts in which they represent a central tenet. For instance, ETs constitute an ideal case study to describe and separate the two components of evolution proposed by Simpson (1944), the tempo and mode in evolution, and their mutually independent variations (Felice et al., 2018; Michaud et al., 2018). The strength of an ET can be quantified as the magnitude of a vector, providing a practical way of representing the speed (or tempo) of evolution. The direction of the same vector represents the mode of evolution (i.e., observed pattern of variation). Phenotypic ETs can also be investigated in the framework of evolutionary landscapes, accounting for variations in evolutionary constraints affecting a clade through time or in response to environmental shifts. Phenotypic ETs were frequently found as the resulting evolutionary outcome in studies about the controversial field of evolutionary predictability, that focuses on the occurrence of repetitive and foreseeable patterns in evolution under specific conditions (e.g., recurrent patterns in insular systems-de Visser \& Krug, 2014; Whittaker et al., 2017). For instance, directional evolution towards a relative reduction of flight muscles in insular colonies of birds was found to be a predictable and frequent phenotypic specialization produced by an ecological release from mammalian predators (Wright et al., 2016). And it is specialisation that was suggested by recent conservation studies to serve as a fundamental proxy for vulnerability and extinction rate increases of species in future or hypothetical scenarios, supporting the idea that research on phenotypic ETs is conceivable to become additional topics included in the future scientific debate on climate change and conservation biology (Gallagher et al., 2015).

Our main goal is to propose a modern theoretical framework for the study of phenotypic ETs, based on Seilacher's theory of morphodynamics (Briggs, 2017; Seilacher \& Gishlick, 2015), that explores the interactions between ETs and the main evolutionary factors involved in their occurrence. The theory of morphodynamics can be visually represented with a tetrahedron whose nodes correspond to the main evolutionary factors influencing morphological evolution: phylogenetic history, developmental constraints, environment, and biological function (Briggs, 2017). Thanks to the inclusion of the environmental component, morphodynamics might be considered an expansion of the concept of 'constructional morphology' proposed by Seilacher (1970, 1991), which considered the role of phylogenetic history, developmental constraints, and biological function in determining morphological evolution of organisms. Both morphodynamics and constructional morphology "replace 
the idealistic concept of 'bauplan', in which the concept of organisms as integrated, functional machines was substituted for the sterile notion of organisms as systems of pure morphological form" (Bock, 1991, p. 27).

In order to achieve our goal, after introducing a new operative definition of ETs, our discussion will start by investigating the influence of environment and evo-devo constraints on phenotypic ETs, focusing on the benefits resulting from the adoption of spatially-explicit models and on the role played by the plasticity-first evolution model. Then, we will elaborate on the importance of including phylogenetic information by adopting phylogenetic comparative methods to evaluate evolutionary patterns. Finally, we will describe how recent advances in morphological quantification can be applied to investigate the relationship between (directional) morphological evolution and biological function. In order to suggest new frontiers of research, in the last section we will discuss how, by exploiting the catalysing effect of climate change on evolution, research on directional evolution induced by global change might represent an ideal arena for the validation of hypotheses about the predictability of evolution.

\section{Geometric Definition and Classification of Evolutionary Trends}

The description of an ET, traditionally considered as an unambiguous phenomenon, has often been limited to anecdotal lists including self-evident examples, without any further quantitative consideration (Stayton, 2015b). The absence of a widely-supported operative definition led to the formulation of multiple (and partially overlapping or conflicting) theoretical definitions. Furthermore, this absence contributed to generate confusion about the relationship between evolutionary concepts like ETs, convergence, divergent evolution, and parallelism, that were considered nested or independent evolutionary cases according to different authors (Manceau et al., 2010; Scotland, 2011).

The concept of '(non) parallel evolution' (i.e., continuum from convergent through parallel to divergent evolution) was recently proposed by Bolnick et al. (2018) to describe the independent evolution of replicate populations, in reference to bacterial cultures of laboratory experiments. The aim of this term is to adopt a pattern-based (Box 1), geometric, and quantitative definition to include in a single concept all classes of evolutionary patterns existing whenever two or more replicates evolving toward a constant direction in a trait space are taken into account. According to this approach, the distinction between parallelism, convergence, and divergent evolution entirely relies on the amplitude of the angles among these evolutionary trajectories, represented as vectors in the trait space. The transposition of (non) parallel evolution to the macroevolutionary scale (Fig. 1) represents a perfect geometric definition of ETs. This definition constitutes a key innovation to solve the main theoretical issue related to the study of ETs, that is the description of the existing classes of ETs (and their relationships) occurring whenever multiple clades are simultaneously considered (Stayton, 2015a). Three possible classes (i.e., parallel, convergent, and divergent evolution) are conceivable from this perspective. The criterion of distinction between them relies, even in this case, on the orientation of the evolutionary trajectories shown by the single groups (Fig. 1B-D). Therefore, parallelism will be the resulting outcome when evolutionary trajectories point in the same direction, otherwise, a condition of convergence or divergent evolution will occur, depending on whether trajectories respectively point 'at' or 'away from' a region of the trait space.

This operative definition of ETs is in line with the idea of 'directional evolution' provided by Hunt and Carrano (2010). The authors described directional evolution, Brownian Motion (BM) evolution, and stasis as the most relevant models in long-term (i.e., paleontological) macroevolutionary studies, even stating that only mean and variance of the distribution of trait values matter over paleontological temporal scales. In particular, Hunt and Carrano (2010) distinguished between directional and $\mathrm{BM}$ evolution arguing that whenever deviations from the mean of this distribution occur, the resulting model will be defined as directional evolution; otherwise, we will be in presence of $\mathrm{BM}$ evolution. Coherently with this distinction, several methods using BM as a null hypothesis were proposed in order to detect specific ETs, such as convergent evolution (e.g., Wheatsheaf, $\mathrm{C} 1$ and $\theta$ metrics-Arbuckle et al., 2014; Stayton, 2015a; Castiglione et al., 2019a) and directional trends through time (e.g., Castiglione et al., 2019b; Sherratt et al., 2016). Nevertheless, in the presence of constraints, BM-like diffusions might result in a directional pattern of evolution determined by the position of constraints in the trait space (Fisher, 1986). For example, the presence of a lower boundary in size evolution has been supposed to be the mechanism that accounts for Cope's rule (i.e., tendency for clades to increase their size through time - Stanley, 1973). According to this vision, Cope's rule is simply produced by an increase in size variance through time (Gould, 1988b). Based on these considerations, McShea (1994, p. 1747) distinguished between passive and driven ETs, stating that, "in a driven trend, the distribution mean increases on account of a force (which may manifest itself as a bias in the direction of change) that acts on lineages throughout the trait space in which diversification occurs. In a passive system, no pervasive force or bias exists, but the mean increases because change in one direction is blocked by a boundary, or other inhomogeneity, in some limited region of the trait space". McShea also validated the occurrence of driven and passive ETs in the 
Fig. 1 Scatterplots resulting from the occurrence of any possible class of ETs considering species (black dots) belonging to one (A) or multiple (B-D) clades (ellipses) at the same time and relative examples from the literature. (A) Directionality in limb evolution of fossil equids. (B) Convergent evolution in body shapes of marine vertebrates, such as penguins, dolphins, and ichthyosaurs. (C) Divergent evolution in morphology of Darwin's finches. (D) Parallel evolution produced by the occurrence of Allen's rule in body shape of ursids and canids. Evolutionary trajectories are represented as vectors (red arrows) in the trait space (Color figure online)

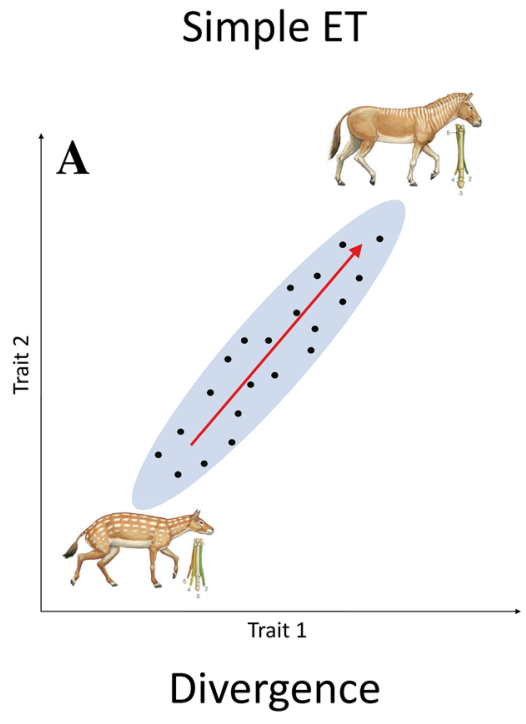

\section{Convergence}
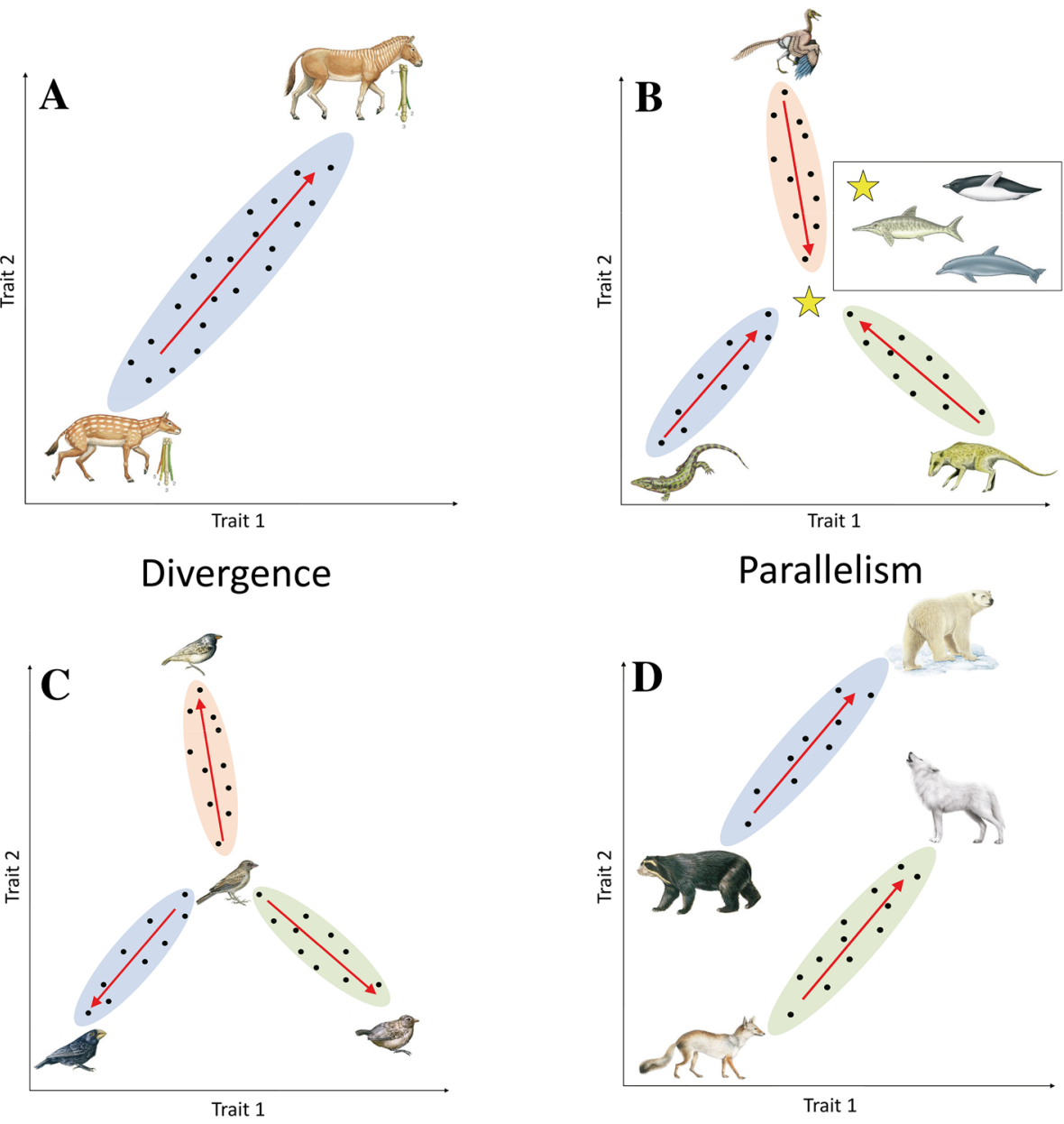

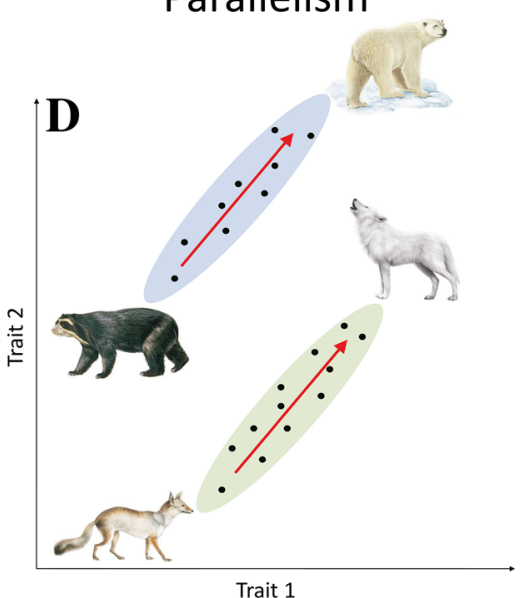

evolution of horses, brachiopods, and rodents, and tried to classify as driven or passive trends several other live hypotheses, such as trends through time in size, complexity, and energy intensiveness (McShea, 1998). Our operative definition of ETs can be applied to both passive and driven ETs.

According to our definition of ETs, parallel evolution, convergence, and divergent evolution can be considered as patterns produced whenever, analysing multiple clades at the same time, a portion of the groups undergoes an episode of directional evolution, whereas other groups are subject to a different evolutionary regime. The adoption of a multiscale approach is therefore recommended to recognise these classes of ETs. Performing evolutionary analyses at different taxonomic levels has also been suggested as a promising avenue to define the evolutionary boundaries acting on a clade more rigorously and the relationships between morphology, evolutionary tempo, and ecological divergence in a broad comparative context (Tamagnini et al., 2017). In this sense, research on ETs would take a major step forward if a pattern-based approach, like the one described above (that can only validate or disprove the presence of a pattern), was systematically accompanied by multifactorial analyses. These analyses would also allow researchers to take into account data relative to underpinning dynamics leading to the occurrence of directional evolution (e.g., ecological variables or life-history traits). When it comes to evolutionary dynamics occurring in phenotypic evolution, the tangled nature of interactions between the factors underpinning morphological evolution has already been explored by the theory of morphodynamics (Briggs, 2017, pp. 203-204), concluding that four components have a predominant role: phylogenetic history, developmental constraints, environment, and biological function. However, the central role of genetic constraints (e.g., scarce genetic variation, low mutation rate, and multivariate genetic correlations) was subsequently recognized as a limiting factor for phenotypic evolution (Futuyma, 2010). Given the role of evo-devo processes as a bridge between genetic and developmental constraints (Müller, 2007), it is in our opinion advisable to adopt them as the fourth factor included in the present theoretical framework (Fig. 2), replacing the factor 'developmental constraints' originally included in the theory of morphodynamics. In 
the next sections, we will explore the interactions between phenotypic ETs and these evolutionary factors (starting with evo-devo constraints and environment, then shifting onto phylogeny and finally focusing on biological function in the light of new perspectives in morphological quantification), also mentioning applications or methodological approaches that can be used in these contexts.

\section{Box 1}

\section{Process-Based Versus Pattern-Based Approaches and Implications with Evo-Devo Mechanisms}

The necessity to identify an underpinning evolutionary process to validate the presence of evolutionary patterns has been criticised and gave rise to a long-standing debate in the history of evolutionary biology (Mayr, 1956). When it comes to ETs, the emerging solutions to this dispute are all stemming from two alternative approaches to individuate the presence of a phenomenon that led to the formulation of process-based and patternbased definitions. Process-based definitions require the $a$ priori demonstration that a common mechanism is acting in response to the same evolutionary conditions in all considered groups. By contrast, pattern-based definitions bind the validation of an evolutionary pattern to the mere fulfilment of mathematical or geometrical criteria, generally based on ratios or angular values: it follows that this type of approach is always coupled with quantitative measures and null models (Rosenblum et al., 2014; Stayton, 2015a).

The improved understanding of the functioning of evodevo mechanisms under different evolutionary conditions initiated an identification process of the linkage between phenotypic variation and specific evo-devo constraints. For instance, Felice and colleagues (2018) hypothesised that phenotypic integration and modularity act as a bridge between developmental, genetic, and functional levels of trait associations, influencing the morphology of organisms, even catalyzing the evolution and the production of biodiversity. Under suitable conditions, phenotypic integration canalises trait variations along limited evolutionary directions, meeting one of the preconditions to the rise of an ET (Goswami et al., 2014; Wagner \& Zhang, 2011). Variations in consolidated patterns of integration between morphological modules were also observed to play an essential role in the iterative occurrence of extreme phenotypes, like the skull of saber-toothed cats, classifiable as episodes of convergent evolution (Meloro \& Slater, 2012). The recent advances in mechanism investigation might tempt evolutionary biologists to adopt process-based definitions of ETs in every situation, formulating a developmental or physiological explanation for an observed directionality simply assuming its validity on the basis of different and previously observed case studies. However, despite this type of definitions frequently represents the optimal choice in microevolution, the validity of evo-devo processes in broad comparative data still has to be extensively proved, and process-based approaches were frequently demonstrated to produce contradictions or misinterpretations in the explanation of evolutionary patterns when considering large taxonomic scales (Felice et al., 2018; Meiri, 2011; Stayton, 2015a). Furthermore, process-based definitions systematically fail to categorize the occurrence of a specific pattern, like the presence of directionality, whenever an evolutionary mechanism in place cannot be easily found (e.g., Collar et al., 2014).
Fig. 2 Visualisation of the theoretical framework for the study of phenotypic ETs, including the main evolutionary factors (rectangles), processes (rhombi), methods (ellipses), and respective interactions (arrows) described in the present work. Coloured elements represent the key elements of morphodynamics (Color figure online)

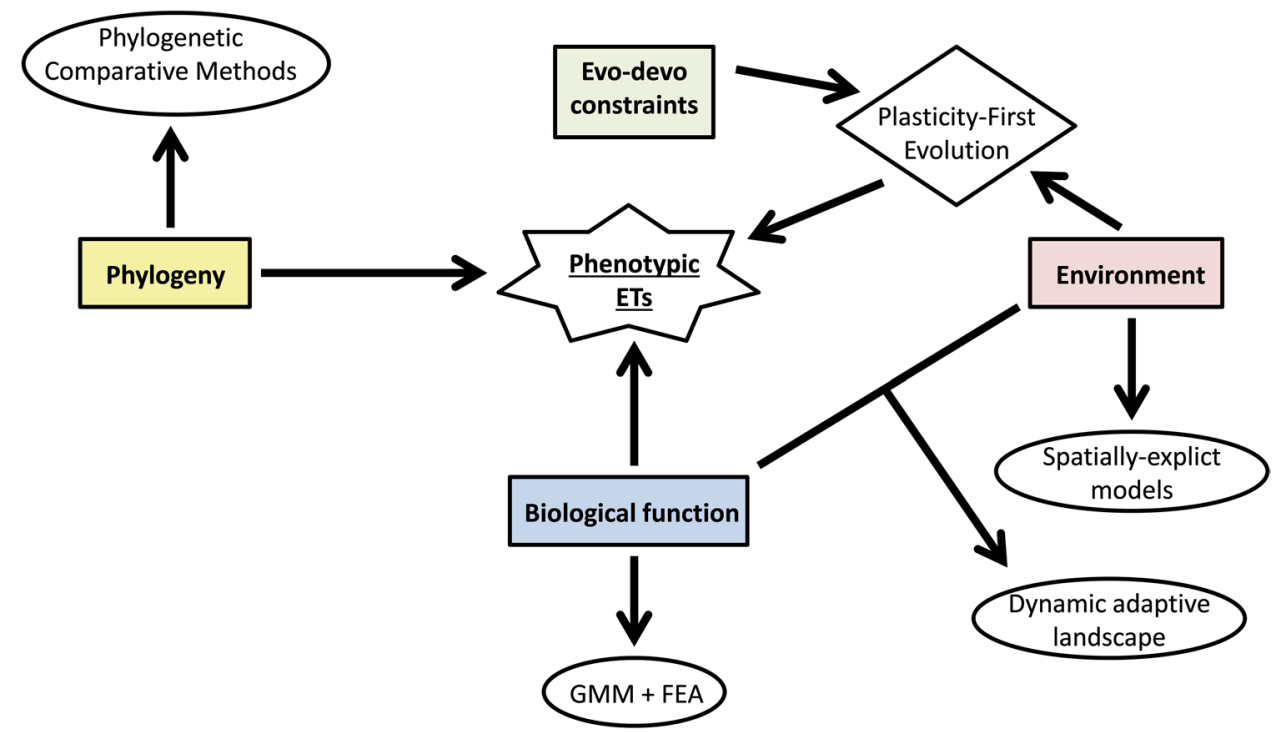


The advantage of adopting pattern-based definitions in macroevolution partially resides in their extreme flexibility, allowing researchers to accommodate additional modifiers easily and clearly define nested cases within an evolutionary phenomenon (Stayton, 2015a). But more importantly, pattern-based validations are suitable for making inferences about different systems in broad comparative data and can serve to steer post hoc studies on the mechanisms responsible for the observed patterns. Inferences based on a process-based approach would require, by contrast, an a priori validation of the putatively involved mechanism for each different system, unless resulting in gross extrapolations beyond the conditions of the system (e.g., Menge, 1992). Obtaining this sort of process corroboration is challenging due to its experimental nature, which limits the number and the scale of analysable samples. The necessity to shift from small scale understanding to large scale predictions is shared in other fields of evolutionary biology, like macroecology, where there is an increasing demand to identify general, predictive, and empirical relationships in natural systems (Currie, 2019).

\section{The Role of Evo-Devo Constraints and Environment in Shaping Directional Macroevolutionary Patterns and Processes: Plasticity-First Evolution and Spatially-Explicit Models}

Investigating the interconnection between evo-devo constraints, environment, and phenotypic variations is crucial for better understanding macroevolutionary processes and improving the prediction of patterns produced by evolutionary directionality. The recent formulation of the evolutionary mechanism named plasticity-first evolution (PFE) model (Levis \& Pfennig, 2016) has emphasised the influence of environment and its interactions with evo-devo constraints in shaping phenotypes. According to the PFE model, the environmentally initiated phenotypic change, known as phenotypic plasticity, acts as a precursor of evolutionary adaptation. The environmental variability uncovers the cryptic genetic variation shaping the extant phenotypic plasticity. Then, genetic accommodation leads to the adaptive refinement of favoured phenotypes whenever a condition of diffused polyphenism (i.e., condition where different phenotypes can easily coexist) is not favoured by selection. Shifting the focus on the resulting macroevolutionary effects, directional and persistent environmental stimuli might underpin the rise of an ET acting on preexisting phenotypic plasticity. Despite concerns regarding the PFE model being raised due to its (at least apparently) partial inconsistency with the modern synthesis of evolutionary biology (i.e., phenotypic plasticity acting in specific cases as a precursor of evolutionary adaptation), leading to define it as a mere artefact of lab studies (Ho \& Zhang, 2018), Levis and colleagues (2018) recently validated the presence of PFE in North American spadefoot toads of the genus Spea demonstrating that a persistent environmental stimulus (i.e., presence of different preys and competitors) caused the evolution of intraspecific dietinduced plasticity into fixed morphs within the genus.

Leaving aside the uncertainties relative to the underlying evolutionary mechanisms, the focus on geographical and ecological information in a theoretical model of directional evolutionary patterns recently became a central topic in macroevolution. For instance, Polly (2018) highlighted that existing statistical models used to study evolutionary patterns rely on a Fisherian view of evolution, whereby species are considered single panmictic populations (i.e., populations in which all individuals are potential partners) whose traits are the same regardless of spatial and environmental influences (Fisher, 1930). According to this vision, evolutionary outcomes disregard drift and habitat-specific selection (and are likely to be affected by a misjudgement of the impact of sexual selection). However, both drift and habitat-specific selection might produce, under neutral evolution, phenotypic paths of evolution similar to those resulting from the occurrence of directionality under Fisherian conditions, like the rise of morphological gradients along species distribution ranges (e.g., cranial shape evolution of European common shrews-Polly, 2018). To this aim, Polly also used computational modelling to produce simulations, supporting the idea that drift and habitat-specific selection represent a potential source of misinterpretations in macroevolution. The inclusion of spatially structured variation in evolutionary models (i.e., Wrightian view of evolution) would allow considering metapopulations composed by interacting demes characterised by different trait values: this would enable the distinction between patterns produced by spatial processes, like drift and habitat-specific selection, and ETs associated with non-neutral and directional evolutionary regimes (Hanski, 1999). A potential solution would be the development of individual-based macroevolutionary models. However, a major obstacle to go in this direction is the discrepancy between the lineagebased focus, typical of evolutionary theories, and the attention for individual organisms and their interactions over timescales of a few generations, which often belongs to ecology (Rosindell et al., 2015). In spite of the temporary absence of available spatially explicit models to distinguish between Fisherian and Wrightian trait evolution, ecological models based on the neutral theory of biodiversity represents a key tool to bridge the gap between individualbased ecology and macroevolution, as well as the inclusion 
of spatial gradients of selection, metacommunity spatial dynamics, and trait distributions of fossil record (Badgley \& Finarelli, 2013; Lyons \& Smith, 2013; Maestri et al., 2018; Polly, 2018).

\section{Phylogenetic Comparative Methods and the Necessity to Adopt a Phylogenetically-Informed Approach for the Study of Phenotypic ETs}

Macroevolutionary datasets should be collected following a rigorous sampling design. However, evolutionary biologists face issues related to accessibility, incompleteness or sample size, often leading to the implementation of simplified sampling designs that suffer from unknown biases (Albert et al., 2010). Considering taxonomically related groups of species that occur together in space, instead of exclusively analysing monophyletic groups, is often referred to as assemblage approach and is a widespread practice in evolutionary biology, specifically for the study of phenotypic ETs (Meiri, 2011; Stroud et al., 2015). This approach shows, whenever applied to evolutionary biology, the same shortcomings occurring in its original field of application, ecology. For instance, Mittelbach and Schemske (2015) highlighted that assemblagebased analyses can be misleading without a preliminary recognition of the dynamic nature of species pools and the processes underlying species pool formation. The resulting almost inevitable ineffectiveness to describe evolutionary dynamism over large spatial and/or temporal scales originates discrepancies frequently found in assemblage-based studies on ETs (e.g., inconsistent presence of Bergmann's rule in different assemblages of the mammalian order Carnivora-Diniz-Filho et al. 2007, 2009) and suggests to always adopt a phylogenetically-informed approach for the study of macroevolutionary patterns and processes, specifically relying on phylogenetic comparative methods (PCMs).

PCMs are statistical models that estimate the evolutionary regime that best approximates the tempo and mode of evolution acting on the considered traits, allowing researchers to correct for biases due to the non-independence of sampled observations in macroevolutionary samples (i.e., phylogenetic relationships - Felsenstein, 1985; Venditti et al., 2011). Although discussing existing PCMs and their assumptions is beyond the aim of the present work, the existing literature about this topic is already impressive (e.g., Adams \& Collyer, 2019; Cooper et al., 2016 and references therein). Taking into account paleontological data, the possibility to include the available fossil evidence in phylogenetic reconstructions was recently proved to improve estimations of the best model of evolution, facilitating the detection of changes in tempo and mode of evolution and the reconstruction of the ancestral states, preventing, therefore, erroneous inferences and increasing the statistical power of PCMs (Mitchell et al., 2019; Schnitzler et al., 2017). The inclusion of paleontological datasets in complex modelling of temporal and spatial evolutionary patterns and the application of PCMs to ecological information represent a promising way to proceed for evolutionary biologists in the foreseeable future and would facilitate the detection of ETs and their variations in strength and direction through time (Castiglione et al., 2019a, 2019b; Maestri et al., 2018).

\section{New Perspectives in Morphological Quantification: Evolutionary Landscapes, Geometric Morphometrics, and Finite Element Analysis}

The tangled interactions between variations in selective advantage deriving from biological function and phenotypic or genotypic responses have been traditionally rendered using evolutionary landscapes since this type of graph is meant to show differences in the level of selective advantage intrinsic to a specific set of trait values (Arnold et al., 2001; Wright, 1932). Nowadays, several versions of evolutionary landscapes are available in phenotypic evolution to investigate these interactions both at clade and single-lineage level, enabling, for instance, to recognize the occurrence of evolution driven by ecological opportunity (Hunt et al., 2015; Caetano and Harmon, 2017; Voje, 2020). Regardless of the adopted version of landscape, these graphs are particularly indicated for the visual detection of ETs, since their conformation is likely to reflect the acting evolutionary pattern. For instance, in the absence of boundaries (i.e., driven ETs sensu McShea, 1994), favoured phenotypes are distributed along a linear line whenever directionality is common to the entire sample. The resulting evolutionary landscape is a rectilinear ridge (Fig. 3A). Parallelism, convergence and divergent evolution originate a series of parallel ridges, a peak or a depression into the landscapes (Fig. 3B-D), respectively. By contrast, non-directional evolution, as in the case of BM evolution, is likely to generate a flat surface (Fig. 3E). In the presence of boundaries (i.e., passive ETs sensu McShea, 1994), their position in the evolutionary landscape contributes to determining the resulting evolutionary pattern.

Recently developed techniques of morphological quantifications (like geometric morphometrics-GMM - and finite element analysis-FEA) allow researchers to quantitatively describe different aspects of phenotypic variation such as size, shape, and functional performance. However, these techniques produce highly multivariate data generally difficult to be implemented for an adequate evolutionary landscape or to 


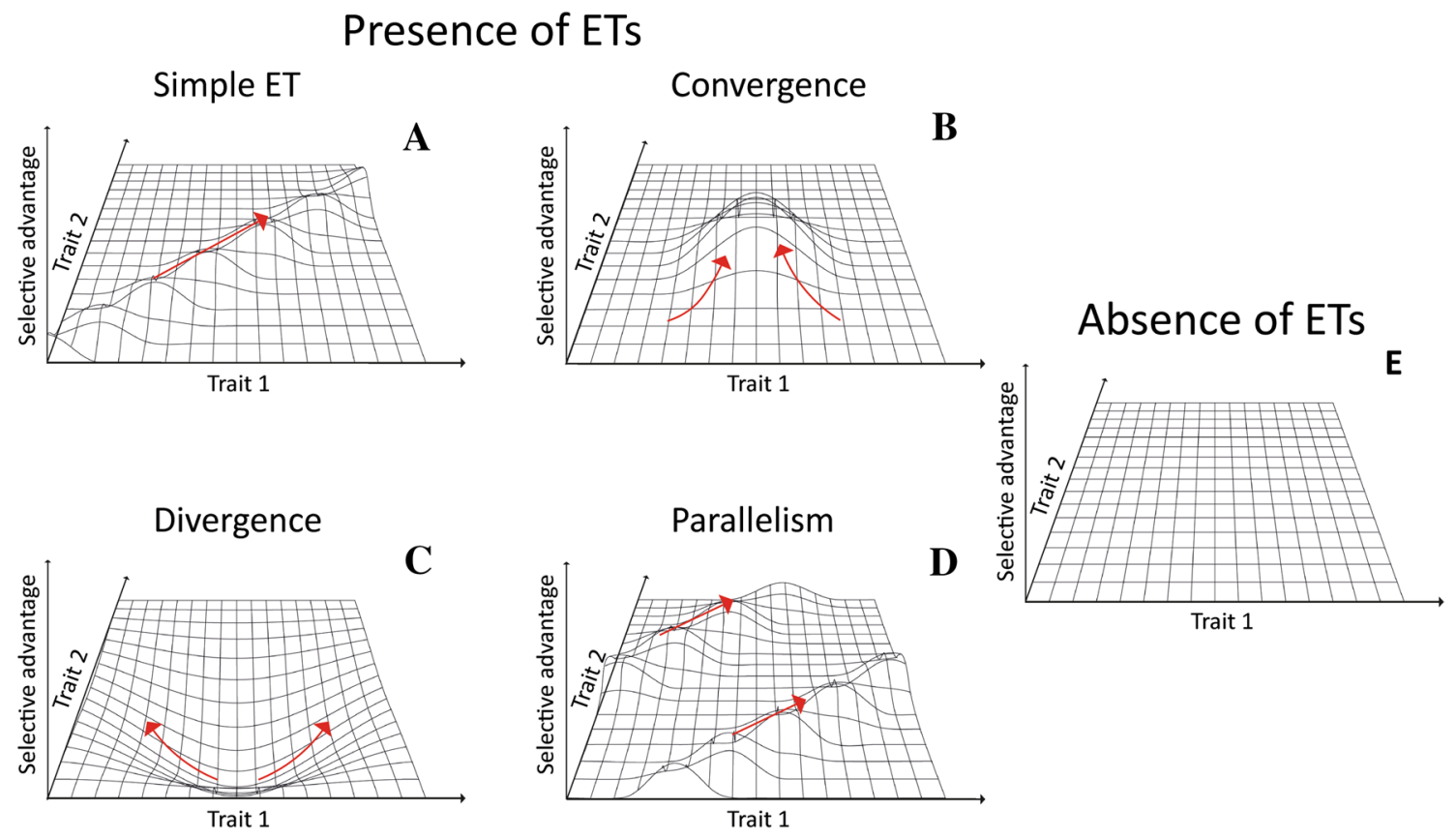

Fig. 3 Examples of evolutionary landscapes resulting, in the absence of boundaries, from the presence of ETs in one (A) or multiple (B-D) clades or from the absence of ETs (E). Simple ETs are linked to a rectilinear ridge configuration (A). Convergent evolution is associated with a peak in the landscape (B), whereas divergence and parallel-

be corrected applying PCMs. For instance, Adams and Collyer (2018a) recently demonstrated that the most common methods (i.e., log-likelihood or AIC score-based techniques) used to estimate the best evolutionary model in multivariate GMM analyses become more ill-conditioned as the ratio between considered morphological traits and sampled species increases or as considered models become more complex. Furthermore, paleomorphological studies have to face issues linked to the scarcity and the fragmented nature of preserved material, plus distortions of fossils produced by taphonomic processes (Arbour \& Currie, 2012). To overcome these issues, a new generation of PCMs is rising in GMM, allowing operators to ground estimations of the best model and its parameters on methods unbiased by an increase in trait dimensionality (i.e., statistical tests relying on traces of covariance matricesAdams \& Collyer, 2018a), to adopt innovative techniques of randomizing residuals in phylogenetic ANOVAs and regression models (Adams \& Collyer, 2018b) or to graphically visualise potential trends in phylogenetic signal (i.e., phylogenetically aligned component analysis-Collyer \& Adams, 2020). Furthermore, recent studies demonstrated how FEA analyses can be employed, relying on trait spaces derived from GMM, to obtain quantitative surfaces describing the performances of different morphologies (O'Higgins et al., 2011; Polly et al., 2016). The combination of multiple performance surfaces (e.g., optimisation concepts such as Pareto front) represents ism are linked to depressions (C) and parallel ridges (D), respectively. Flat surfaces (E) are a typical landscape configuration representing the absence of ETs, as in case of Brownian Motion evolution. Red arrows represent most likely trajectories of directional evolution (Color figure online)

an informative tool for the construction of phenotypic evolutionary landscapes that show optimal morphologies able to contemporarily carry out all functions associated with the selected performances (see Jones et al., 2021 for a worked example about the synapsid-mammal locomotor transition). Combining these techniques of morphological quantification was also proved to be useful for retrodeforming altered fossil specimens or reconstructing damaged remains, as well as for hypothesising and analysing non-available intermediate morphologies (Gunz et al., 2009; Schlager et al., 2018; Tseng, 2013). The possibility to consider non-preserved or purely hypothetical evolutionary forms allows researchers to investigate the occurrence of phenotypic ETs filling unexplored regions of morphological trait spaces, obtaining highly detailed landscapes and characterising the response of functional performance produced by different types of continuous variables, ranging from ecological to physiological ones. A further step forward in eco-evolution is the concept of dynamic adaptive landscape proposed by Laughlin and Messier (2015), whose primary focus is detecting changes occurring in an evolutionary landscape along environmental gradients. This technique uses multivariate trait distributions to determine functional trade-offs at different taxonomic scales, improving the understanding of functional trait evolution and its link to the environmental conditions, and therefore represents a powerful tool for studying the interactions between 
biological function and environment. Adopting a dynamic version of phenotypic evolutionary landscapes (potentially also including paleontological evidence) seems to be a promising target, on the one hand, to visualize selective advantage variations along stratigraphic sequences and, on the other, to graphically show the initial outbreak, the constant transformations, and the final weakening of an ET through time (Fig. 4).
The Search for Phenotypic Evolutionary Trends in Climate Change Scenarios as a Tool for Understanding the Predictability of Evolution

As discussed above, pattern-based methods accompanied by multifactorial approaches deriving from a morphodynamicsbased theoretical framework will pave the way to new potential applications for studies on phenotypic ETs. Research on ETs in the context of global changes can represent a timely and promising endeavour, particularly considering the predictability of evolution.

Clarifying under what conditions evolution proceeds along predictable pathways would enable researchers to increase and refine the use of evolutionary biology in several applied contexts, for instance, providing tools for
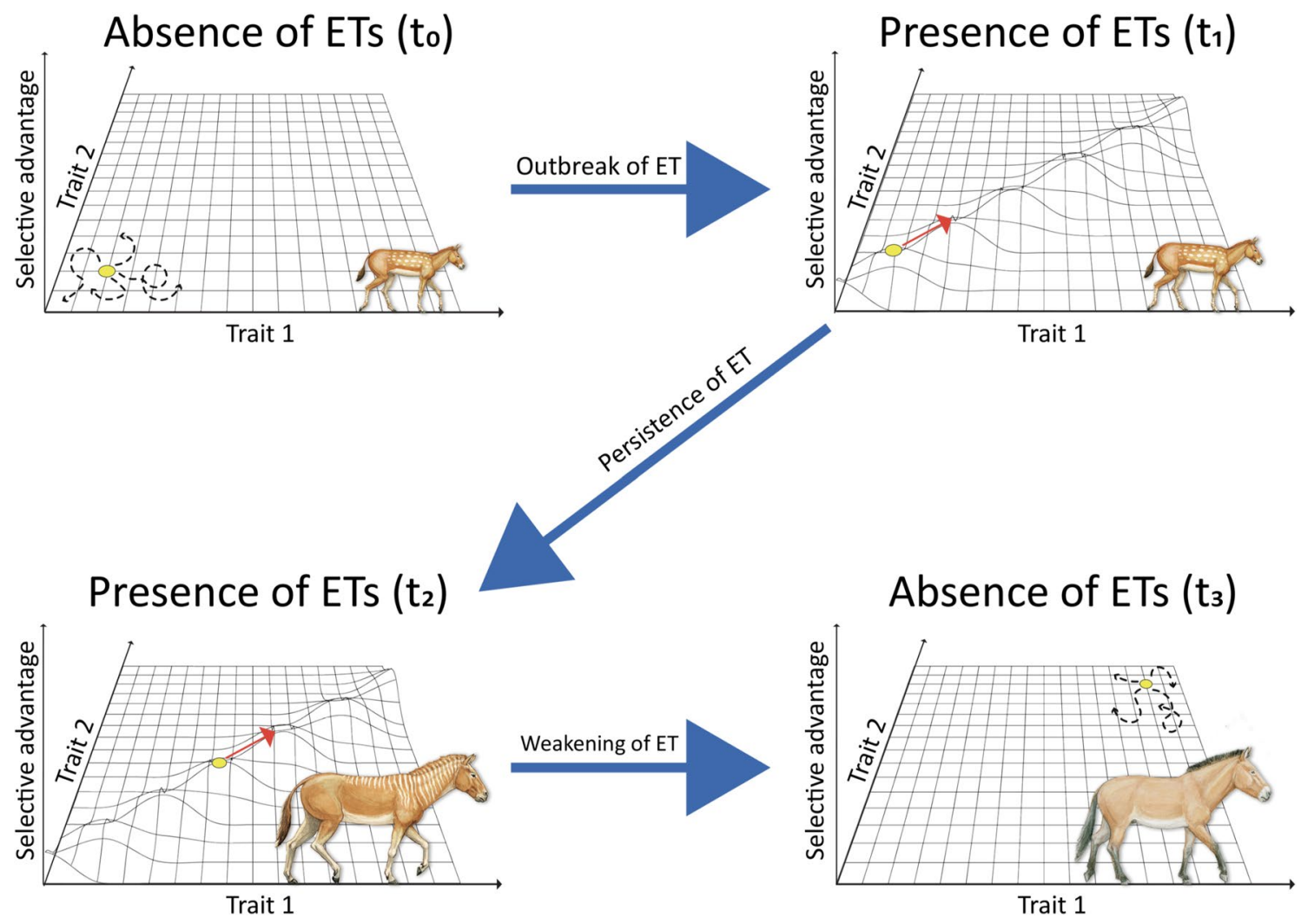

Fig. 4 Example of dynamic evolutionary landscape including the case study of fossil and living equids. A typical scenario for the occurrence of ETs is represented by an initial condition of null selective advantage differences within a given trait space (that is likely to result in Brownian Motion evolution and a flat evolutionary landscape $-\mathrm{t}_{0}$ ) that is disrupted by variations in an external factor (e.g., environmental changes). These variations might lead to a temporary phase of directional evolution (potentially reflected by changes in the configuration of the evolutionary landscape $-t_{1}$ and $t_{2}$ ). The disappearance of the external forcing can terminate this phase (e.g., end of environmental changes and return of a flat evolutionary land- scape configuration $-t_{3}$ ). A similar evolutionary scenario might have occurred in limb evolution of equids: the environmental transition from forests to grasslands started during the late Oligocene is likely to have induced an event of directional evolution towards the acquisition of the monodactyl posture within this clade. Before and after this phase, the absence of ETs (e.g., Brownian Motion evolution) can be considered a likely evolutionary outcome, even in the light of the presence of more stable environmental conditions. Yellow dots represent different species of equids that occurred at different (Color figure online) 
sustainability assessments and reproducibility of laboratory experiments (Rego-Costa et al., 2018). The first pioneering research in this field found strong sources of stochasticity, as erratic fluctuations of the environment or genetic drift, as limiting factors of evolutionary predictability (Sæther \& Engen, 2015). By contrast, investigating the importance of deterministic chaos under simulated repetitive evolutionary conditions, Rego-Costa and colleagues (2018) recently pointed out that a strong forcing by a changing environment (e.g., rapid shifts in selective optima) can improve the predictability of eco-evolutionary dynamics, otherwise resulting in a chaotic scenario under constant evolutionary conditions. It follows that climate change events, whenever causing directional shifts of selective conditions, might represent an enhancer of evolutionary predictability.

The current phase of anthropogenic climate change, resulting in a pattern of diffused global warming, is threatening the existing biodiversity and is potentially leading the entire planet towards a sixth mass extinction that might require millions of years to restore comparable levels of species richness (Barnosky et al., 2011; Davis et al., 2018; Rosenzweig et al., 2008). These environmental changes force species to shift their geographical distribution, change their phenology or alternatively develop adaptations in their morphology and/or physiology relying on an admixture of genetic change and plasticity (Bellard et al., 2012; Hoffmann \& Sgrò, 2011). The rise of a phenotypic ET, frequently regarding variations in organism size or shape and changes in the integration between biological modules, constitutes a frequent and highly repeatable event induced by climate change, probably because reflecting a phenotypic response to a constant and directional change in the environment that fits a linear model (MacLean et al., 2018 and references therein). For this reason, research on the occurrence of phenotypic ETs in currently changing environments has the potential to become, in the near future, an indispensable tool for clarifying the dynamics underpinning evolutionary predictability, allowing researchers to verify hypotheses (by the exploitation of the catalysing effect of climate change on evolution) that would otherwise require geological times to be empirically validated in more stable environments. For this purpose, metrics measuring evolvability (i.e., capacity of a system to produce evolutionary adaptations - Colegrave \& Collins, 2008; Kirschner \& Gerhart, 1998) might be used to assess the likelihood of a clade to develop phenotypic ETs in changing environments. An investigation of morphological integration in the cranium of the mammalian order Carnivora revealed, for instance, that canids possess a higher amount of phenotypic evolvability if compared to other carnivorans. This outcome is mainly produced by an elevated incidence of pure repetitive sequences, promoting new genetic variants by duplication (Laidlaw et al., 2007; Machado et al., 2018). The conspicuous evolvability of this family was suggested to have underpinned several episodes of directionality in cranial evolution of canids associated with dietary shifts (e.g., increase in relative brachyrostry in hypercarnivore species-Machado et al., 2018). Recent macroevolutionary analyses performed on avian morphology suggest the presence of strong form-function associations that channel phenotypic variation enhancing evolvability towards specific directions of the trait space and produce repeated patterns of morphological convergence that result in highly predictable phenotypes (Felice et al., 2018; Pigot et al., 2020). This evidence seems to confirm the potential that studies on phenotypic ETs in climate change contexts might have for understanding the dynamics underpinning the predictability of evolution. Such studies might open the way for innovative approaches that might improve several inferences about patterns and processes in macroevolution, even producing indirect repercussions in climate change and conservation biology.

\section{Concluding Remarks and Future Directions}

ETs channel trait variations and often occur in the presence of directional shifts of selective conditions. Relying on the orientation of evolutionary trajectories in trait spaces, ETs involving multiple clades can be seen as a continuum that goes from convergent through parallel to divergent evolution, making multiscale analyses necessary to distinguish these classes.

Since phylogenetic history, evo-devo constraints, environment, and biological function all play a fundamental role in the occurrence of phenotypic ETs, adopting a morphodynamics-based approach to research on ETs in phenotypic evolution represent a pivotal step forward to fully understand the dynamics that produce this type of evolutionary patterns. Therefore, using methods to assess the influence of each of these factors taken individually, or even performing multifactorial analyses, is of vital importance to shed light on this field (Fig. 5). The possibility to incorporate spatially structured variations, together with the inclusion of fossil record in already existing modelling of evolution, is expected to produce the next major breakthroughs, in terms of analyses on phenotypic ETs, that are likely to be designed in the foreseeable future. An intriguing new frontier of macroevolution is represented by the possibility to perform research on ETs in the presence of extreme environmental shifts, like those resulting from the current phase of climate change. Such research might be crucial for refining predictions of future or hypothetical evolutionary outcomes and might lead to indirect implications in climate change and conservation biology. 


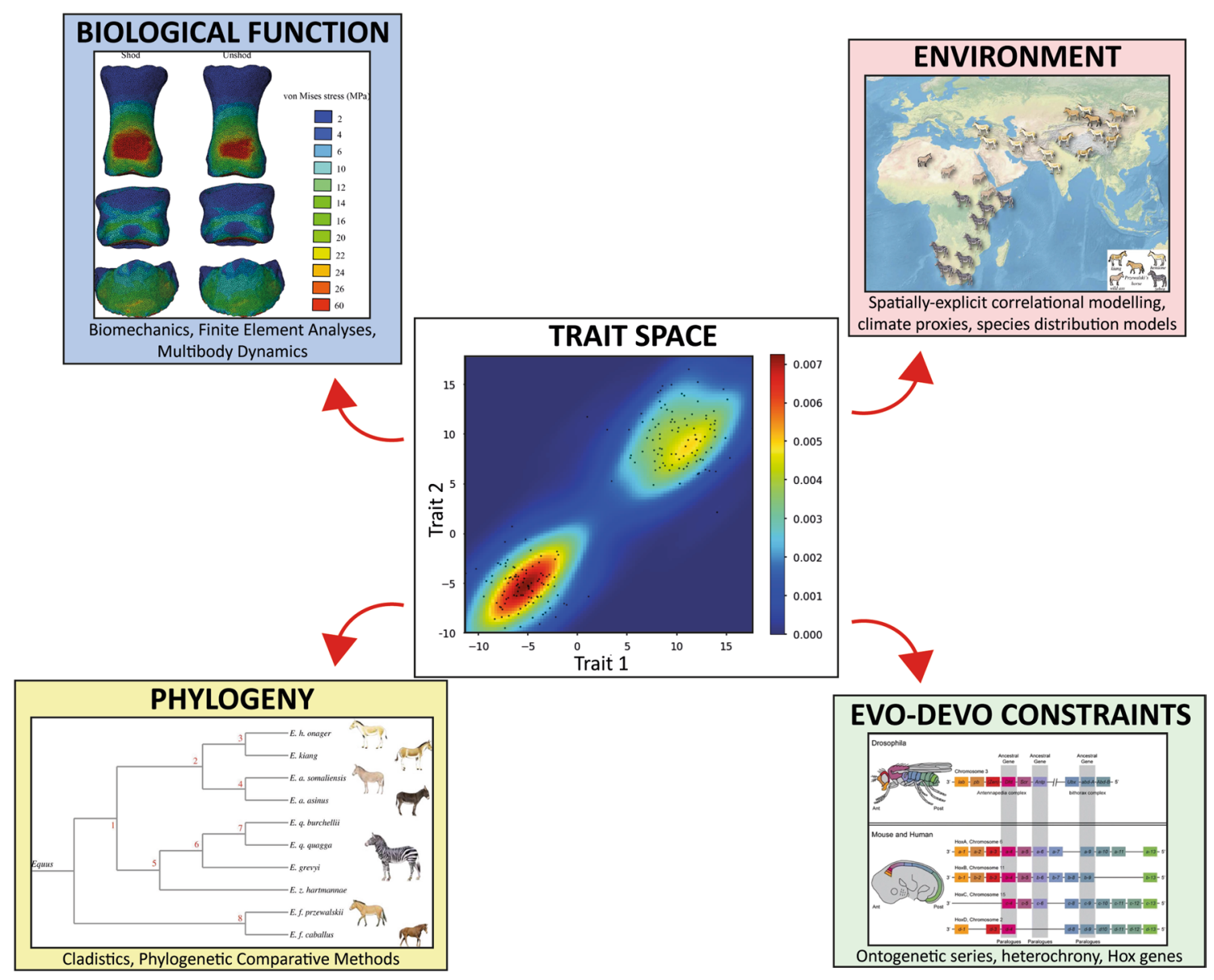

Fig. 5 Operational framework to investigate ETs using morphodynamics. Any trend observed plotting phenotypic traits in a trait space can be investigated in the light of four factors (i.e., phylogeny, evodevo constraints, environment, and biological function). The impact of each factor is mapped back into the trait space to quantify the amount of selective advantage variation explained over the phenotypic distribution. Optimisation concepts such as Pareto front can be employed to account for multiple constraints and functions within the same surface. Pareto front was applied to phenotypic evolution mainly to investigate the limits of biological function. Typical examples can be found in Shoval et al. (2012), where the authors demonstrated that phenotypic distribution over a morphological trait space generally falls within a triangle whose vertices represent the archetype (i.e., optimal morphological solution for a particular task). Sho-

\section{Glossary}

Morphodynamics

Conceptual framework used to describe all processes influencing a morphology during its evolutionary and developmental histories, including also the impact of external environment.

val et al. also demonstrated that this concept can be applied to gene expression in the bacterium $E$. coli. Other recent examples still cover constraints in biological function, such as Polly et al. (2016), Dickson and Pierce (2019), and Jones et al. (2021). Interestingly, Polly (2020) demonstrated that functional optimization can also be interpreted applying PCMs, thus covering both biological function and phylogeny within the same framework. The visualisation of horse FEA (light blue box) was modified from Panagiotopoulou et al. (2016), both the spatial distribution plot and the phylogeny of extant equids (pink and yellow boxes, respectively) were modified from Cucchi et al. (2017), and the visualisation of Hox genes in Drosophila, mice, and humans (green box) was modified from Pang and Thompson (2011). Trait space plot (white box) was produced following http:// qingkaikong.blogspot.com/2018/05/ (Color figure online)

Evolutionary developmental biology

(Evo-devo) 
Modularity

Integration

Cryptic genetic variation

Genetic accommodation

Ecological opportunity

Geometric Morphometrics (GMM)

Finite Element Analysis (FEA)

Deterministic chaos Phenology
Condition where a biological structure is subdivided into units (i.e., modules) that possess a strong internal covariation but weakly interact with the other units. In morphology, a classic example of modular structure is the mammalian skull, traditionally subdivided into facial and neurocranial modules.

Tendency of multiple traits to covary throughout a biological structure.

Alternative developmental variants uncovering genetic variations that have negligible effects on phenotypic variations except under atypical conditions.

Evolutionary process that refines a phenotype towards an adaptive condition through selection driving quantitative genetic changes.

Condition where a lineage or a clade can suddenly occupy a new niche space with a wide range of underexploited resources. The sudden disappearance of competitors, the access to new environments or the appearance of key innovations are some of the most common triggers producing ecological opportunity.

Technique of morphological quantification able to extract (and separate) size and shape data from a biological structure, relying on the Cartesian coordinates of corresponding anatomical points, known as landmarks, placed on every specimen of the analysed sample.

Engineering approach frequently employed to quantify the morphological function of a biological structure. The results, expressed in terms of stress and strain, allow estimating the effects produced by applying different loads to the structure and the resulting deformation.

Dependency of the dynamics of a system on its initial conditions.

Timing of life cycle events of a species, such as flowering, fruiting, and seasonal migrations.
Acknowledgements We are deeply grateful to David Polly and Markus Bastir for their feedback about preliminary versions or presentations regarding this work. This research received support from the SYNTHESYS programme (ES-TAF-2750 awarded to DT) and the "Avvio alla Ricerca" funds provided by the University of Rome "La Sapienza" (awarded to DT). The research was also supported by a grant from the Italian Ministry of Education, University and Research (PRIN project 2017KLZ3MA) to LM and DC.

Funding Open access funding provided by Università degli Studi di Roma La Sapienza within the CRUI-CARE Agreement. This research received support from the SYNTHESYS programme (ES-TAF-2750 awarded to DT) and the "Avvio alla Ricerca" funds provided by the University of Rome "La Sapienza" (awarded to DT). The research was also supported by a grant from the Italian Ministry of Education, University and Research (PRIN project 2017KLZ3MA) to LM and DT.

Data availability Not applicable.

Code availability Not applicable.

\section{Declarations}

Conflict of interest The authors declare that they have no conflict of interest.

Open Access This article is licensed under a Creative Commons Attribution 4.0 International License, which permits use, sharing, adaptation, distribution and reproduction in any medium or format, as long as you give appropriate credit to the original author(s) and the source, provide a link to the Creative Commons licence, and indicate if changes were made. The images or other third party material in this article are included in the article's Creative Commons licence, unless indicated otherwise in a credit line to the material. If material is not included in the article's Creative Commons licence and your intended use is not permitted by statutory regulation or exceeds the permitted use, you will need to obtain permission directly from the copyright holder. To view a copy of this licence, visit http://creativecommons.org/licenses/by/4.0/.

\section{References}

Adams, D. C., \& Collyer, M. L. (2018a). Multivariate phylogenetic comparative methods: Evaluations, comparisons, and recommendations. Systematic Biology, 67(1), 14-31.

Adams, D. C., \& Collyer, M. L. (2018b). Phylogenetic ANOVA: Group-clade aggregation, biological challenges, and a refined permutation procedure. Evolution, 72(6), 1204-1215.

Adams, D. C., \& Collyer, M. L. (2019). Phylogenetic comparative methods and the evolution of multivariate phenotypes. Annual Review of Ecology, Evolution, and Systematics, 50(1), 405-425.

Albert, C. H., Yoccoz, N. G., Edwards, T. C., Graham, C. H., Zimmermann, N. E., \& Thuiller, W. (2010). Sampling in ecology and evolution - bridging the gap between theory and practice. Ecography, 33(6), 1028-1037.

Allen, J. A. (1877). The Influence of physical conditions in the genesis of species.

Alroy, J. (2000). Understanding the dynamics of trends within evolving lineages. Paleobiology, 26(3), 319-329.

Arbour, V. M., \& Currie, P. J. (2012). Analyzing taphonomic deformation of ankylosaur skulls using retrodeformation and finite element analysis. PLoS ONE, 7(6), e39323. 
Arbuckle, K., Bennett, C. M., \& Speed, M. P. (2014). A simple measure of the strength of convergent evolution. Methods in Ecology and Evolution, 5(7), 685-693.

Arnold, S. J., Pfrender, M. E., \& Jones, A. G. (2001). The adaptive landscape as a conceptual bridge between micro- and macroevolution. Genetica, 112(1), 9-32.

Ayala, F. J. (1988). Can 'progress' be defined as a biological concept? In M. H. Nitecki (Ed.), Evolutionary progress (pp. 75-96). University of Chicago Press.

Badgley, C., \& Finarelli, J. A. (2013). Diversity dynamics of mammals in relation to tectonic and climatic history: Comparison of three Neogene records from North America. Paleobiology, 39(3), 373-399.

Barnosky, A. D., Matzke, N., Tomiya, S., Wogan, G. O. U., Swartz, B., Quental, T. B., Marshall, C., McGuire, J. L., Lindsey, E. L., Maguire, K. C., Mersey, B., \& Ferrer, E. A. (2011). Has the Earth's sixth mass extinction already arrived? Nature, 471(7336), 51-57.

Bellard, C., Bertelsmeier, C., Leadley, P., Thuiller, W., \& Courchamp, F. (2012). Impacts of climate change on the future of biodiversity. Ecology Letters, 15(4), 365-377.

Bergmann, C. (1847). Über die verhältnisse der wärmeökonomie der thiere zu ihrer größe.

Bock, W. J. (1991). Explanations in Konstruktionsmorphologie and evolutionary morphology. In N. Schmidt-Kittler \& K. Vogel (Eds.), Constructional morphology and evolution (pp. 9-29). Springer.

Bolnick, D. I., Barrett, R. D. H., Oke, K. B., Rennison, D. J., \& Stuart, Y. E. (2018). (Non)parallel evolution. Annual Review of Ecology, Evolution, and Systematics, 49(1), 303-330.

Bowler, P. J. (1977). Edward drinker cope and the changing structure of evolutionary theory. Isis, 68(2), 249-265.

Briggs, D. E. G. (2017). Seilacher, konstruktions-morphologie, morphodynamics, and the evolution of form. Journal of Experimental Zoology Part B: Molecular and Developmental Evolution, 328(3), 197-206.

Caetano, D. S., \& Harmon, L. J. (2017). Ratematrix: An R package for studying evolutionary integration among several traits on phylogenetic trees. Methods in Ecology and Evolution, $1920-1927$

Cardini, A. (2019). Craniofacial allometry is a rule in evolutionary radiations of placentals. Evolutionary Biology, 46(3), 239-248.

Cardini, A., \& Polly, P. D. (2013). Larger mammals have longer faces because of size-related constraints on skull form. Nature Communications, 4(1), 1-7.

Castiglione, S., Serio, C., Tamagnini, D., Melchionna, M., Mondanaro, A., Febbraro, M. D., Profico, A., Piras, P., Barattolo, F., \& Raia, P. (2019). A new, fast method to search for morphological convergence with shape data. PLoS One, 14(12), e0226949.

Castiglione, S., Serio, C., Mondanaro, A., Febbraro, M. D., Profico, A., Girardi, G., \& Raia, P. (2019). Simultaneous detection of macroevolutionary patterns in phenotypic means and rate of change with and within phylogenetic trees including extinct species. PLoS One, 14(1), e0210101.

Colegrave, N., \& Collins, S. (2008). Experimental evolution: Experimental evolution and evolvability. Heredity, 100(5), 464-470.

Collar, D. C., Reece, J. S., Alfaro, M. E., Wainwright, P. C., \& Mehta, R. S. (2014). Imperfect morphological convergence: Variable changes in cranial structures underlie transitions to durophagy in moray eels. The American Naturalist, 183(6), E168-E184.

Collyer, M. L., \& Adams, D. C. (2020). Phylogenetically aligned component analysis. Methods in Ecology and Evolution, 12(2), 359-372.

Cooper, N., Thomas, G. H., \& FitzJohn, R. G. (2016). Shedding light on the 'dark side' of phylogenetic comparative methods. Methods in Ecology and Evolution, 7(6), 693-699.
Cucchi, T., Mohaseb, A., Peigné, S., Debue, K., Orlando, L., \& Mashkour, M. (2017). Detecting taxonomic and phylogenetic signals in equid cheek teeth: Towards new palaeontological and archaeological proxies. Royal Society Open Science, 4(4), 160997.

Currie, D. J. (2019). Where Newton might have taken ecology. Global Ecology and Biogeography, 28(1), 18-27.

Davis, M., Faurby, S., \& Svenning, J.-C. (2018). Mammal diversity will take millions of years to recover from the current biodiversity crisis. Proceedings of the National Academy of Sciences, 115(44), 11262-11267.

Dayan, T., \& Simberloff, D. (1998). Size patterns among competitors: Ecological character displacement and character release in mammals, with special reference to island populations. Mammal Review, 28(3), 99-124.

de Visser, J. A. G. M., \& Krug, J. (2014). Empirical fitness landscapes and the predictability of evolution. Nature Reviews Genetics, 15(7), 480-490.

Dickson, B. V., \& Pierce, S. E. (2019). Functional performance of turtle humerus shape across an ecological adaptive landscape. Evolution, 73(6), 1265-1277.

Diniz-Filho, J. A. F., Bini, L. M., Rodríguez, M. Á., Rangel, T. F. L. V. B., \& Hawkins, B. A. (2007). Seeing the forest for the trees: Partitioning ecological and phylogenetic components of Bergmann's rule in European Carnivora. Ecography, 30(4), 598-608.

Diniz-Filho, J. A. F., Rodríguez, M. Á., Bini, L. M., Olalla-Tarraga, M. Á., Cardillo, M., Nabout, J. C., Hortal, J.-N., \& Hawkins, B. A. (2009). Climate history, human impacts and global body size of Carnivora (Mammalia: Eutheria) at multiple evolutionary scales. Journal of Biogeography, 36(12), 2222-2236.

Felice, R. N., Randau, M., \& Goswami, A. (2018). A fly in a tube: Macroevolutionary expectations for integrated phenotypes. Evolution, 72(12), 2580-2594.

Felsenstein, J. (1985). Phylogenies and the comparative method. The American Naturalist, 125(1), 1-15.

Fisher, R. A. (1930). The genetical theory of natural selection. Clarendon Press.

Fisher, D. C. (1986). Progress in organismal design. In D. M. Raup \& D. Jablonski (Eds.), Patterns and processes in the history of life (pp. 99-117). Springer.

Futuyma, D. J. (2010). Evolutionary constraint and ecological consequences. Evolution, 64(7), 1865-1884.

Gallagher, A. J., Hammerschlag, N., Cooke, S. J., Costa, D. P., \& Irschick, D. J. (2015). Evolutionary theory as a tool for predicting extinction risk. Trends in Ecology and Evolution, 30(2), 61-65.

Goswami, A., Smaers, J. B., Soligo, C., \& Polly, P. D. (2014). The macroevolutionary consequences of phenotypic integration: From development to deep time. Philosophical Transactions of the Royal Society B: Biological Sciences, 369(1649), 20130254.

Gould, S. J. (1988a). On replacing the idea of progress with an operational notion of directionality. In M. H. Nitecki (Ed.), Evolutionary progress (pp. 319-338). University of Chicago Press.

Gould, S. J. (1988b). Trends as changes in variance: A new slant on progress and directionality in evolution. Journal of Paleontology, $62(3), 319-329$.

Gould, S. J. (2002). The structure of evolutionary theory. Harvard University Press.

Gregory, T. R. (2008). Evolutionary trends. Evolution: Education and Outreach, 1(3), 259-273.

Gunz, P., Mitteroecker, P., Neubauer, S., Weber, G. W., \& Bookstein, F. L. (2009). Principles for the virtual reconstruction of hominin crania. Journal of Human Evolution, 57(1), 48-62.

Hanski, I. (1999). Metapopulation ecology. OUP Oxford.

Ho, W.-C., \& Zhang, J. (2018). Evolutionary adaptations to new environments generally reverse plastic phenotypic changes. Nature Communications, 9(1), 1-11. 
Hoffmann, A. A., \& Sgrò, C. M. (2011). Climate change and evolutionary adaptation. Nature, 470(7335), 479-485.

Hunt, G., \& Carrano, M. T. (2010). Models and methods for analyzing phenotypic evolution in lineages and clades. Paleontological Society Papers, 16, 245-269.

Hunt, G., Hopkins, M. J., \& Lidgard, S. (2015). Simple versus complex models of trait evolution and stasis as a response to environmental change. Proceedings of the National Academy of Sciences, 112(16), 4885-4890.

Jones, K. E., Dickson, B. V., Angielczyk, K. D., \& Pierce, S. E. (2021). Adaptive landscapes challenge the "lateral-to-sagittal" paradigm for mammalian vertebral evolution. Current Biology. https://doi. org/10.1016/j.cub.2021.02.009

Kirschner, M., \& Gerhart, J. (1998). Evolvability. Proceedings of the National Academy of Sciences, 95(15), 8420-8427.

Kowalewsky, V. O. (1874). Monographie der gattung Anthracotherium Cuv., und versucheiner natürlichen classification der fossilen huftiere. Palaeontographica, 22, 210-285.

Laidlaw, J., Gelfand, Y., Ng, K.-W., Garner, H. R., Ranganathan, R., Benson, G., \& Fondon, J. W. (2007). Elevated basal slippage mutation rates among the Canidae. Journal of Heredity, 98(5), 452-460.

Laughlin, D. C., \& Messier, J. (2015). Fitness of multidimensional phenotypes in dynamic adaptive landscapes. Trends in Ecology and Evolution, 30(8), 487-496.

Levis, N. A., Isdaner, A. J., \& Pfennig, D. W. (2018). Morphological novelty emerges from pre-existing phenotypic plasticity. Nature Ecology and Evolution, 2(8), 1289-1297.

Levis, N. A., \& Pfennig, D. W. (2016). Evaluating 'plasticity-first' evolution in nature: Key criteria and empirical approaches. Trends in Ecology and Evolution, 31(7), 563-574. https://doi.org/10. 1016/j.tree.2016.03.012

Lyons, S. K., \& Smith, F. A. (2013). Macroecological patterns of body size in mammals across time and space. In F. A. Smith \& S. K. Lyons (Eds.), Animal body size: Linking pattern and process across space, time, and taxonomic group (pp. 116-144). University of Chicago Press.

Machado, F. A., Zahn, T. M. G., \& Marroig, G. (2018). Evolution of morphological integration in the skull of Carnivora (Mammalia): Changes in Canidae lead to increased evolutionary potential of facial traits. Evolution, 72(7), 1399-1419.

MacLean, H. J., Nielsen, M. E., Kingsolver, J. G., \& Buckley, L. B. (2018). Using museum specimens to track morphological shifts through climate change. Philosophical Transactions of the Royal Society B: Biological Sciences, 374(1763), 20170404.

Maestri, R., Monteiro, L. R., Fornel, R., de Freitas, T. R. O., \& Patterson, B. D. (2018). Geometric morphometrics meets metacommunity ecology: Environment and lineage distribution affects spatial variation in shape. Ecography, 41(1), 90-100.

Manceau, M., Domingues, V. S., Linnen, C. R., Rosenblum, E. B., \& Hoekstra, H. E. (2010). Convergence in pigmentation at multiple levels: Mutations, genes and function. Philosophical Transactions of the Royal Society B: Biological Sciences, 365(1552), 2439-2450.

Mayr, E. (1956). Geographical character gradients and climatic adaptation. Evolution, 10(1), 105-108.

McKinney, M. L. (1990). Classifying and analysing evolutionary trends. In K. J. McNamara (Ed.), Evolutionary trends (pp. 28-58). University of Arizona Press.

McNamara, K. J. (2006). Evolutionary trends. American Cancer Society.

McShea, D. W. (1994). Mechanisms of large-scale evolutionary trends. Evolution, 48(6), 1747-1763.

McShea, D. W. (1998). Possible largest-scale trends in organismal evolution: Eight "live hypotheses." Annual Review of Ecology and Systematics, 29(1), 293-318.
McShea, D. W. (2005). The evolution of complexity without natural selection, a possible large-scale trend of the fourth kind. Paleobiology, 31(sp5), 146-156.

McShea, D. W., \& Brandon, R. N. (2010). Biology's first law: The tendency for diversity and complexity to increase in evolutionary systems. University of Chicago Press.

McShea, D. W., Wang, S. C., \& Brandon, R. N. (2019). A quantitative formulation of biology's first law. Evolution, 73(6), 1101-1115.

Meiri, S. (2011). Bergmann's rule - what's in a name? Global Ecology and Biogeography, 20(1), 203-207.

Meloro, C., \& Slater, G. J. (2012). Covariation in the skull modules of cats: The challenge of growing saber-like canines. Journal of Vertebrate Paleontology, 32(3), 677-685.

Menge, B. A. (1992). Community regulation: Under what conditions are bottom-up factors important on rocky shores? Ecology, 73(3), $755-765$.

Michaud, M., Veron, G., Peignè, S., Blin, A., \& Fabre, A.-C. (2018). Are phenotypic disparity and rate of morphological evolution correlated with ecological diversity in Carnivora? Biological Journal of the Linnean Society, 124(3), 294-307.

Mitchell, J. S., Etienne, R. S., \& Rabosky, D. L. (2019). Inferring diversification rate variation from phylogenies with fossils. Systematic Biology, 68(1), 1-18.

Mittelbach, G. G., \& Schemske, D. W. (2015). Ecological and evolutionary perspectives on community assembly. Trends in Ecology and Evolution, 30(5), 241-247.

Müller, G. B. (2007). Evo-devo: Extending the evolutionary synthesis. Nature Reviews Genetics, 8(12), 943-949.

O’Higgins, P., Cobb, S. N., Fitton, L. C., Gröning, F., Phillips, R., Liu, J., \& Fagan, M. J. (2011). Combining geometric morphometrics and functional simulation: An emerging toolkit for virtual functional analyses. Journal of Anatomy, 218(1), 3-15.

Panagiotopoulou, O., Rankin, J. W., Gatesy, S. M., \& Hutchinson, J. R. (2016). A preliminary case study of the effect of shoewearing on the biomechanics of a horse's foot. PeerJ, 4, e2164.

Pang, D., \& Thompson, D. N. P. (2011). Embryology and bony malformations of the craniovertebral junction. Child's Nervous System, 27(4), 523-564.

Pigot, A. L., Sheard, C., Miller, E. T., Bregman, T. P., Freeman, B. G., Roll, U., Seddon, N., Trisos, C. H., Weeks, B. C., \& Tobias, J. A. (2020). Macroevolutionary convergence connects morphological form to ecological function in birds. Nature Ecology and Evolution, 4(2), 230-239.

Polly, P. D. (2020). Functional tradeoffs carry phenotypes across the valley of the shadow of death. Integrative and Comparative Biology, 60(5), 1268-1282.

Polly, P. D. (2018). Spatial processes and evolutionary models: A critical review. Palaeontology, 62(2), 175-195.

Polly, P. D., Stayton, C. T., Dumont, E. R., Pierce, S. E., Rayfield, E. J., \& Angielczyk, K. D. (2016). Combining geometric morphometrics and finite element analysis with evolutionary modeling: Towards a synthesis. Journal of Vertebrate Paleontology, 36(4), e1111225.

Raia, P., Carotenuto, F., Mondanaro, A., Castiglione, S., Passaro, F., Saggese, F., Melchionna, M., Serio, C., Alessio, L., Silvestro, D., \& Fortelius, M. (2016). Progress to extinction: Increased specialisation causes the demise of animal clades. Scientific Reports, 6(1), 30965.

Raia, P., \& Fortelius, M. (2013). Cope's law of the unspecialized, Cope's rule, and weak directionality in evolution. Evolutionary Ecology Research, 15(7), 747-756.

Rego-Costa, A., Débarre, F., \& Chevin, L.-M. (2018). Chaos and the (un)predictability of evolution in a changing environment. Evolution, 72(2), 375-385. 
Rosenblum, E. B., Parent, C. E., \& Brandt, E. E. (2014). The molecular basis of phenotypic convergence. Annual Review of Ecology, Evolution, and Systematics, 45(1), 203-226.

Rosenzweig, C., Karoly, D., Vicarelli, M., Neofotis, P., Wu, Q., Casassa, G., Menzel, A., Root, T. L., Estrella, N., Seguin, B., Tryjanowski, P., Liu, C., Rawlins, S., \& Imeson, A. (2008). Attributing physical and biological impacts to anthropogenic climate change. Nature, 453(7193), 353-357.

Rosenzweig, M. L., \& McCord, R. D. (1991). Incumbent replacement: Evidence for long-term evolutionary progress. Paleobiology, 17(3), 202-213.

Rosindell, J., Harmon, L. J., \& Etienne, R. S. (2015). Unifying ecology and macroevolution with individual-based theory. Ecology Letters, 18(5), 472-482.

Sæther, B.-E., \& Engen, S. (2015). The concept of fitness in fluctuating environments. Trends in Ecology and Evolution, 30(5), 273-281.

Schlager, S., Profico, A., Vincenzo, F. D., \& Manzi, G. (2018). Retrodeformation of fossil specimens based on 3D bilateral semilandmarks: Implementation in the R package "Morpho." PLoS One, 13(3), e0194073.

Schnitzler, J., Theis, C., Polly, P. D., \& Eronen, J. T. (2017). Fossils matter - Understanding modes and rates of trait evolution in Musteloidea (Carnivora). Evolutionary Ecology Research, 18(2), 187-200.

Scotland, R. W. (2011). What is parallelism? Evolution and Development, 13(2), 214-227.

Seilacher, A., \& Gishlick, A. D. (2015). Morphodynamics. CRC Press.

Seilacher, A. (1970). Arbeitskonzept zur konstruktions-morphologie. Lethaia, 3(4), 393-396.

Seilacher, A. (1991). Self-organizing mechanisms in morphogenesis and evolution. In N. Schmidt-Kittler \& K. Vogel (Eds.), Constructional morphology and evolution (pp. 251-271). Springer.

Sherratt, E., Alejandrino, A., Kraemer, A. C., Serb, J. M., \& Adams, D. C. (2016). Trends in the sand: Directional evolution in the shell shape of recessing scallops (Bivalvia: Pectinidae). Evolution, 70(9), 2061-2073.

Shoval, O., Sheftel, H., Shinar, G., Hart, Y., Ramote, O., Mayo, A., Dekel, E., \& KavanaghAlon, K. U. (2012). Evolutionary tradeoffs, Pareto optimality, and the geometry of phenotype space. Science, 336(6085), 1157-1160.
Simpson, G. G. (1944). Tempo and mode in evolution. Columbia University Press.

Stanley, S. M. (1973). An explanation for Cope's rule. Evolution, 27(1), $1-26$.

Stayton, C. T. (2015a). The definition, recognition, and interpretation of convergent evolution, and two new measures for quantifying and assessing the significance of convergence. Evolution, 69(8), $2140-2153$

Stayton, C. T. (2015b). What does convergent evolution mean? The interpretation of convergence and its implications in the search for limits to evolution. Interface Focus, 5(6), 20150039.

Stroud, J. T., Bush, M. R., Ladd, M. C., Nowicki, R. J., Shantz, A. A., \& Sweatman, J. (2015). Is a community still a community? Reviewing definitions of key terms in community ecology. Ecology and Evolution, 5(21), 4757-4765.

Tamagnini, D., Meloro, C., \& Cardini, A. (2017). Anyone with a longface? Craniofacial evolutionary allometry (CREA) in a family of short-faced mammals, the Felidae. Evolutionary Biology, 44(4), 476-495.

Tseng, Z. J. (2013). Testing adaptive hypotheses of convergence with functional landscapes: A case study of bone-cracking hypercarnivores. PLoS One, 8(5), e65305.

Venditti, C., Meade, A., \& Pagel, M. (2011). Multiple routes to mammalian diversity. Nature, 479(7373), 393-396.

Voje, K. L. (2020). Testing eco-evolutionary predictions using fossil data: Phyletic evolution following ecological opportunity. Evolution, 74(1), 188-200.

Wagner, G. P., \& Zhang, J. (2011). The pleiotropic structure of the genotype-phenotype map: The evolvability of complex organisms. Nature Reviews Genetics, 12(3), 204-213.

Whittaker, R. J., Fernández-Palacios, J. M., Matthews, T. J., Borregaard, M. K., \& Triantis, K. A. (2017). Island biogeography: Taking the long view of nature's laboratories. Science. https:// doi.org/10.1126/science.aam8326

Wright, S. (1932). The roles of mutation, inbreeding, crossbreeding, and selection in evolution. Proceedings of the Sixth International Congress on Genetics, 1, 356-366.

Wright, N. A., Steadman, D. W., \& Witt, C. C. (2016). Predictable evolution toward flightlessness in volant island birds. Proceedings of the National Academy of Sciences, 113(17), 4765-4770. 